\title{
Some Remarks on the Homotopy-Analysis Method
}

\section{and Series Solutions to the Blasius Equation}

\author{
W. Robin \\ Engineering Mathematics Group \\ Edinburgh Napier University \\ 10 Colinton Road, Edinburgh \\ EH10 5DT, UK \\ b.robin@napier.ac.uk
}

Copyright (C) 2013 W. Robin. This is an open access article distributed under the Creative Commons Attribution License, which permits unrestricted use, distribution, and reproduction in any medium, provided the original work is properly cited.

\begin{abstract}
We present an new angle on the introduction of the homotopy-analysis method of Liao [6]. The homotopy-analysis method is then applied to produce a series solution of the Blasius problem in two different ways and comparison made with the work of Wang [7], Hashim [5] and Ahmad [3]. A standard (Aitken [2]) acceleration technique is applied to improve the main results of the analysis.
\end{abstract}

Mathematics Subject Classification: 30B10; 34A25; 34B08; 34B15

Keywords: Homotopy; Adomian; Blassius; series solutions; Aitken acceleration

\section{Introduction}

There has arisen, in recent years, a number of methods of approach to the solution of non-linear differential equations. Of these methods the decomposition method (DM), associated with the name of G. Adomian [1], and the homotopy-analysis method (HAM), developed in its current form by S.J. Liao [6], have proven both enduring (popular) and successful. The general method of approach of the DM is well described in Adomian's monograph [1] and the relationship between the DM and the HAM is addressed in Liao's book [6]. In what follows, we will review the relationship between the HAM and the DM from a somewhat different angle than usual, by re-deriving the standard solution algorithm (see equations (6) to (11) 
below) simply by modifying the (usually nonlinear) equation to be solved, using the basic arithmetic relationships

to recover, in Section 2, the HAM.

$$
\alpha \times 0=0, \quad \alpha+(-\alpha)=0
$$

Once we have recovered the HAM, we use the solution algorithm to reconsider a certain power-series solution to the Blasius equation. We tackle this problem in two ways: as an iterative solution in Section 3 and, more directly, as an assumed power-series solution in Section 4. This brings us into immediate contact with the work of Wang [7] and Hashim [5]- the iterative solution - and Ahmad [3] - the assumed power-series solution.

The treatment presented here is different from both that of Wang and Hashim the basic equation is treated differently - and Ahmad - the derivation is new.

Further, the refined determination of the critical parameter (see equation (17) below) in the solution of the Blasius problem, an unknown initial condition, is treated differently, with the standard Aitkin sequence-acceleration technique being used to advance the convergence of a derived sequence of values converging on this initial condition. The form of the solution to the Blasius problem- a power-series and a sequence of real numbers - facilitates comparison with previous work.

\section{Reviewing the Homotopy-Analysis Algorithm}

We begin by assuming that we wish to solve the (generally) non-linear differential equation

$$
\hat{\mathrm{N}}[\mathrm{u}(\mathbf{r})]=0
$$

(the operator $\hat{\mathrm{N}}$ is in general a non-linear operator) to find the unknown function $\mathrm{u}(\mathbf{r}), \mathbf{r} \in \Omega$, subject to given boundary conditions, as an infinite series of functions of the form

$$
\mathrm{u}(\mathbf{r})=\sum_{\mathrm{k}=0}^{\infty} \mathrm{u}_{\mathrm{k}}(\mathbf{r})
$$

The problem becomes, now, the determination of the functions $\left\{\mathrm{u}_{\mathrm{k}}(\mathbf{r})\right\}_{\mathrm{k}=0}^{\infty}$.

First, we introduce a 'convenience function', $\varepsilon(\mathbf{r}) \neq 0$, by simple multiplication by rewriting the original equation as

$$
\varepsilon(\mathbf{r}) \hat{\mathrm{N}}[\mathrm{u}(\mathbf{r})]=0
$$

and, in addition, we impose a linear term to both sides of the scaled equation, thus 


$$
\hat{\mathrm{L}}[\mathrm{u}(\mathbf{r})]=\hat{\mathrm{L}}[\mathrm{u}(\mathbf{r})]+\varepsilon(\mathbf{r}) \hat{\mathrm{N}}[\mathrm{u}(\mathbf{r})]
$$

where $\mathrm{u}_{0}(\mathbf{r})$ satisfies the boundary conditions and the linear operator $\hat{\mathrm{L}}$ is restrained by

$$
\hat{\mathrm{L}}\left[\mathrm{u}_{0}(\mathbf{r})\right]=0
$$

It is to the modified equation (5) that we now apply Adomian's methodology [1].

First, an ordering parameter, $\lambda$, is introduced, with the equation being written now as

$$
\hat{\mathrm{L}}[\mathrm{u}(\mathbf{r})]=\lambda(\hat{\mathrm{L}}[\mathrm{u}(\mathbf{r})]+\varepsilon(\mathbf{r}) \hat{\mathrm{N}}[\mathrm{u}(\mathbf{r})])
$$

(which, as we see presently, is directly related Liao's homotopy-analysis approach [6]) and the solution, $u(\mathbf{r})$, is expressed as a formal power series in $\lambda$ of the form

$$
\mathrm{u}(\mathbf{r})=\sum_{\mathrm{k}=0}^{\infty} \mathrm{u}_{\mathrm{k}}(\mathbf{r}) \lambda^{\mathrm{k}}
$$

Next, we substitute the formal power series into the parameterised equation, (7), to get

$$
\sum_{\mathrm{k}=0}^{\infty} \hat{\mathrm{L}}\left[\mathrm{u}_{\mathrm{k}}(\mathbf{r})\right] \lambda^{\mathrm{k}}=\lambda\left(\sum_{\mathrm{k}=0}^{\infty} \hat{\mathrm{L}}\left[\mathrm{u}_{\mathrm{k}}(\mathbf{r}) \lambda^{\mathrm{k}}\right]+\varepsilon(\mathbf{r}) \hat{\mathrm{N}}\left[\sum_{\mathrm{k}=0}^{\infty} \mathrm{u}_{\mathrm{k}}(\mathbf{r}) \lambda^{\mathrm{k}}\right]\right)
$$

It is now possible to produce an iterative process, to determine the $\mathrm{u}_{\mathrm{k}}(\mathbf{r})$, from this last relation as follows. Differentiate through the above equation $n+1$ times with respect to $\lambda$, divide through by $(n+1)$ ! and set $\lambda=0$, to get

$$
\hat{\mathrm{L}}\left[\mathrm{u}_{\mathrm{n}+1}\right]=\hat{\mathrm{L}}\left[\mathrm{u}_{\mathrm{n}}\right]+\varepsilon(\mathbf{r}) \mathrm{A}_{\mathrm{n}}\left(\mathrm{u}_{0}, \mathrm{u}_{1}, \mathrm{u}_{2}, \ldots, \mathrm{u}_{\mathrm{n}}\right)
$$

where

$$
A_{n}\left(u_{0}, u_{1}, u_{2}, \ldots, u_{n}\right)=\left.\frac{1}{n !} \frac{d^{n}}{d \lambda^{n}}\left(\hat{N}\left[\sum_{k=0}^{\infty} u_{k}(r) \lambda^{k}\right]\right)\right|_{\lambda=0}
$$

is a so-called Adomian polynomial [1].

The basic equations, (6) and (7), are simply a form of the homotopy-analysis approach of Liao [6]. Indeed, if we combine (6) and (7) and re-arrange them slightly, we end up with

$$
(1-\lambda) \hat{\mathrm{L}}\left[\mathrm{u}(\mathbf{r})-\mathrm{u}_{0}(\mathbf{r})\right]=\lambda \varepsilon(\mathbf{r}) \hat{\mathrm{N}}[\mathrm{u}(\mathbf{r})]
$$

which is essentially the basic starting point of Liao's method [6[, along with equation (8). Indeed, from (12) and (8), we see that with $\lambda=0$ we get 
$\hat{\mathrm{L}}\left[\mathrm{u}_{0}(\mathbf{r})\right]=0$, while with $\lambda=1$ we regain the original equation, that is, equation (2), the basic idea of the homotopy method. Strangely enough, in this approach to the topic, this is now a (micro-) theorem!

The algorithm described by equations (6) to (11), then, is just Liao's homotopy-analysis approach [6]. Clearly, we are free to choose the linear operator $\hat{\mathrm{L}}$ and the 'convenience function' $\varepsilon(\mathbf{r})$, subject to the above limitations on $\mathrm{u}_{0}(\mathbf{r})$, and it is this flexibility that we should try to take advantage of in applications. To see this more clearly, we attack a standard benchmark problem, in the form of the Blasius equation [4].

\section{The Blasius Equation}

As a 'benchmark' example of the method, we consider the Blasius equation [4]

$$
f^{\prime \prime \prime}(\eta)+f(\eta) f^{\prime \prime}(\eta)=0
$$

with the boundary conditions

$$
f(\eta)=f^{\prime}(\eta)=0, f^{\prime}(\eta) \rightarrow 1 \text { as } \eta \rightarrow \infty
$$

Using the Crocco-Wang substitutions (see Wang [7])

$$
y=f^{\prime \prime}(\eta) \text { and } x=f^{\prime}(\eta)
$$

the Blasius boundary value problem transforms into (the dashes denote differentiation with respect to $\mathrm{x}$ )

$$
\left\{\begin{array}{c}
y(x) y^{\prime \prime}(x)+x=0, \quad 0<x<1 \\
y^{\prime}(0)=0, \quad y(1)=0
\end{array}\right.
$$

which is a (singular) boundary value problem of reduced order. Further, from $f^{\prime}(\eta)=0, x=f^{\prime}(\eta)$ and $y=f^{\prime \prime}(\eta)$, we see that

$$
\mathrm{f}^{\prime \prime}(0)=\mathrm{y}(0)=\mathrm{a}
$$

with 'a' an unknown initial condition (to be determined below) for the original Blasius problem.

The methodology behind the solution process is now straightforward [7]. First, we solve the initial value problem

$$
\left\{\begin{array}{c}
y(x) y^{\prime \prime}(x)+x=0, \quad 0<x<1 \\
y(0)=a, \quad y^{\prime}(0)=0
\end{array}\right.
$$


To get a solution in terms of the unknown constant ' $a$ '. Then, we determine the constant ' $a$ ' by applying the remaining boundary condition, $y(1)=0$.

For this problem we choose the elementary linear differential operator as $\hat{\mathrm{L}} \equiv \frac{\mathrm{d}^{2}}{\mathrm{dx}^{2}}$, with the corresponding elementary differential equation

$$
\hat{\mathrm{L}}\left[\mathrm{y}_{0}(\mathrm{x})\right] \equiv \frac{\mathrm{d}^{2} \mathrm{y}_{0}(\mathrm{x})}{\mathrm{dx}^{2}}=0
$$

with solution $\mathrm{y}_{0}(\mathrm{x})=\mathrm{a}$, with ' $\mathrm{a}$ ' our constant, which satisfies both initial conditions, $\mathrm{y}_{0}(0)=\mathrm{a}$ and $\mathrm{y}_{0}^{\prime}(0)=0$. Our new equation is then (taking $\varepsilon(\mathbf{r})=\varepsilon$ constant)

$$
\mathrm{y}^{\prime \prime}=\lambda\left[\mathrm{y}^{\prime \prime}+\varepsilon\left(\mathrm{y} \mathrm{y}^{\prime \prime}+\mathrm{x}\right)\right]
$$

with the corresponding initial conditions

$$
\mathrm{y}(0)=\mathrm{a}, \quad \mathrm{y}^{\prime}(0)=0
$$

and the assumed solution becomes

$$
y(x)=\sum_{k=0}^{\infty} y_{k}(x) \lambda^{k}
$$

with $\mathrm{y}_{0}(\mathrm{x})=\mathrm{a}$.

Applying the method in this case leads and the following recursive system of linear differential equations $\left(\mathrm{y}_{0}(\mathrm{x})=\mathrm{a}\right)$

$$
\mathrm{y}_{\mathrm{n}+1}^{\prime \prime}=\mathrm{y}_{\mathrm{n}}^{\prime \prime}+\varepsilon \sum_{\mathrm{k}=0}^{\mathrm{n}} \mathrm{y}_{\mathrm{k}} \mathrm{y}_{\mathrm{n}-\mathrm{k}}^{\prime \prime}+\varepsilon \delta_{\mathrm{n} 0} \mathrm{x}, \quad \mathrm{n}=0,1,2,3, \ldots
$$

with

$$
\delta_{\mathrm{n} 0}= \begin{cases}1, & \mathrm{n}=0 \\ 0, & \mathrm{n} \neq 0\end{cases}
$$

which, assuming the choice $\varepsilon=-\frac{1}{\mathrm{a}}$, result in the following approximate solution to (18): 


$$
\begin{aligned}
y(x) \approx a-\frac{x^{3}}{6 a} & -\frac{x^{6}}{180 a^{3}}-\frac{x^{9}}{2160 a^{5}}-\frac{x^{12}}{19008 a^{7}} \\
& -\frac{2099 x^{15}}{299376000 a^{9}}-\frac{31453 x^{18}}{30536352000 a^{11}}-\frac{46061 x^{21}}{285005952000 a^{13}} \\
& -\frac{62749793 x^{24}}{2359849282560000 a^{15}}-\frac{2118841819 x^{27}}{467250157946880000 a^{17}}-\cdots
\end{aligned}
$$

plus seven more terms, omitted for brevity (though see Section 4).

It is necessary, then, to identify the missing initial condition

$$
\mathrm{f}^{\prime \prime}(0)=\mathrm{y}(0)=\mathrm{a}
$$

This is achieved [7] by applying the second boundary condition, $y(1)=0$, to our approximate solution to obtain a polynomial equation for ' $a$ '. The result of applying the condition $\mathrm{y}(1)=0$, at each level of approximation, is a slowly converging sequence of values of ' $a$ ', presented in Table 1. On applying the standard Aitken acceleration technique to the sequence of values of ' $a$ ', we get an 'improved' value of 'a' as

$$
\mathrm{f}^{\prime \prime}(0)=\mathrm{y}(0)=\mathrm{a} \approx 0.468554
$$

$\mathrm{a} \approx 0.468554$ is a difference of about $-0.22 \%$ from the 'accepted' value of 0.469600 .

Hashim [5], improving on the original work of Wang [7], has solved equation (18) using the 'basic' Adomian method. However, Hashim re-arranged the equation and solved the problem

$$
\left\{\begin{array}{c}
y^{\prime \prime}(x)+\frac{x}{y(x)}=0, \quad 0<x<1 \\
y(0)=a, \quad y^{\prime}(0)=0
\end{array}\right.
$$

subject to the remaining boundary condition, $y(1)=0$. Hashim [5] stopped the iteration process at $\mathrm{x}^{21}$, obtaining the first eight terms in (25), and employed Pade' approximants to improve the value of ' $a$ '. to 0.466799 , compared to 0.4669494712 from Table 1 .

\section{A Direct Power-Series Solution}

If, following Ahmad [3], we assume a power-series solution to (18), then we may take 


$$
\mathrm{y}_{\mathrm{k}}(\mathrm{x})=\mathrm{c}_{\mathrm{k}} \mathrm{x}^{\mathrm{k}}
$$

with the coefficients, $\left\{\mathrm{c}_{\mathrm{k}}\right\}_{\mathrm{k}=0}^{\infty}$, constants. If we substitute $\mathrm{y}_{\mathrm{k}}(\mathrm{x})=\mathrm{c}_{\mathrm{k}} \mathrm{x}^{\mathrm{k}}$ into our recursion relation

$$
\mathrm{y}_{\mathrm{n}+1}^{\prime \prime}=\mathrm{y}_{\mathrm{n}}^{\prime \prime}+\varepsilon \sum_{\mathrm{k}=0}^{\mathrm{n}} \mathrm{y}_{\mathrm{k}} \mathrm{y}_{\mathrm{n}-\mathrm{k}}^{\prime \prime}+\varepsilon \delta_{\mathrm{n} 0} \mathrm{x}, \quad \mathrm{n}=0,1,2,3, \ldots
$$

then we would expect to be able to obtain the recurrence relation(s) for the $\left\{\mathrm{c}_{\mathrm{k}}\right\}_{\mathrm{k}=0}^{\infty}$. First, though, we make a few observation.

With $\mathrm{y}_{0}=\mathrm{a}$ and $\varepsilon=-\frac{1}{\mathrm{a}}$, we have, trivially, $\mathrm{y}_{1}=-\frac{\mathrm{x}^{3}}{6 \mathrm{a}}$ and expression (29) simplifies to

$$
\mathrm{y}_{\mathrm{n}+1}^{\prime \prime}=-\frac{1}{\mathrm{a}} \sum_{\mathrm{k}=1}^{\mathrm{n}} \mathrm{y}_{\mathrm{k}} \mathrm{y}_{\mathrm{n}-\mathrm{k}}^{\prime \prime}, \quad \mathrm{n}=1,2,3, \ldots
$$

Assuming now that $\mathrm{y}_{\mathrm{k}}(\mathrm{x})=\mathrm{c}_{\mathrm{k}} \mathrm{x}^{\mathrm{k}}, \mathrm{y}_{0}=\mathrm{a}$ and $\mathrm{y}_{1}=-\frac{\mathrm{x}^{3}}{6 \mathrm{a}}$, we have

$$
\mathrm{c}_{0}=\mathrm{a} \text { and } \mathrm{c}_{1}=-\frac{1}{6 \mathrm{a}}
$$

and the identity

$$
\sum_{n=1}^{\infty}(n+1) n c_{n+1} x^{n-1}=-\frac{1}{a} \sum_{n=1}^{\infty} \sum_{k=1}^{n}(n-k-1)(n-k) c_{k} c_{n-k} x^{n-2}
$$

or

$$
\sum_{n=2}^{\infty}(n+1) n c_{n+1} x^{n-1}+2 c_{2}=-\frac{1}{a} \sum_{n=2}^{\infty} \sum_{k=1}^{n-1}(n-k)(n-k+1) c_{k} c_{n-k+1} x^{n-1}
$$

which leads to

$$
\mathrm{c}_{2}=0
$$

and the recurrence relation

$$
(\mathrm{n}+1) n \mathrm{c}_{\mathrm{n}+1}=-\frac{1}{\mathrm{a}} \sum_{\mathrm{k}=1}^{\mathrm{n}-1}(\mathrm{n}-\mathrm{k})(\mathrm{n}-\mathrm{k}+1) \mathrm{c}_{\mathrm{k}} \mathrm{c}_{\mathrm{n}-\mathrm{k}+1}, \mathrm{n} \geq 2
$$

At this point we have recovered the results of Ahmad [3], who tackled the transformed Blasius problem (18) directly, by assuming a power-series expansion 


$$
\mathrm{y}=\sum_{\mathrm{k}=0}^{\infty} \mathrm{c}_{\mathrm{k}} \mathrm{x}^{\mathrm{k}}
$$

for its solution. In fact, the power-series expansion gives identical results to the 'Adomian approach' of section 2 above (which is hardly surprising). Continuing the power-series expansion calculations up to $x^{246}$, we find an ' $a$ ' value of $\mathrm{a} \approx 0.4693034010$ or, just over $0.6 \%$ difference from the 'accepted' value of 0.469600. Again, we may 'accelerate' the sequence of ' $a$ ' values if required, however, Ahmad [3] has taken the series solution well beyond $x^{246}$ and we refer to Ahmad's Table 1 for further refinement of the value of ' $a$ ' [3].

Ahmad [3] makes a number of points that emerge from the series solution to the transformed Blasius problem. First, after the first two terms, the series progresses in powers of three. This leads to a general expression for the equation evaluating ' $a$ ', that is (in our notation)

$$
\mathrm{a}+\sum_{\mathrm{k}=1}^{\infty} \frac{\mathrm{c}_{3 \mathrm{k}}}{\mathrm{a}^{2 \mathrm{k}-1}}=0
$$

Secondly, the sequence of values that arises from each finite partial sum of the above equation is very slowly converging. This is obvious from the results quoted above also: we go from 0.4674694086 for $\mathrm{x}^{48}$, to $\mathrm{a} \approx 0.4693034010$ for $\mathrm{x}^{246}$.

Finally, there being no error analysis with this general approach to the Blasius equation, Ahmad [3] has developed a second series solution in $\frac{1}{y}$ and shown how this may be used with the original series, for $y$, to produce upper (y-series solution) and lower ( $\frac{1}{y}$-series solution $)$ bounds for ' $a$ '.

\section{Final Remarks}

The approach to the HAM and the classical Blasius problem presented here, differs in a number of ways from previous authors undertakings [3,5,6,7]. First, the development of the HAM is presented as a matter of basic arithmetic, with the insertion of the convenience parameter, $\varepsilon$, and the linear operator, $\hat{\mathrm{L}}$, being purely a matter of multiplication and addition. Secondly, the manner in which the convenience parameter, $\varepsilon$, has been utilised differs from its previous interpretation as a convergence factor [6]. It is this difference in interpretation of the convenience parameter, $\varepsilon$, that has enabled us first to apply the HAM to the Blasius problem developed as (18) - directly and not after a rewrite as (28) - and then to achieve a simplification with the power-series method, having 
circumvented the step of multiplying two infinite series together that would otherwise have arisen, as in Ahmad's approach [3]. Thirdly, the resultssummarized in Table 1 suggest that the Aitkin method [2] used to accelerating the 'a'-sequence as well as being arithmetically straightforward also looks to be reasonably successful, certainly over the range of values studied here.

\section{References}

[1] Adomian G. : Solving Frontier Problems of Physics: The Decomposition Method, Kluwer Academic Publishers, Dordrecht, 1994.

[2] Aitken A.: On Bernoulli's numerical solution of algebraic equations, Proc. Roy. Soc. Edinburgh 46 (1926) 289-305.

[3] Ahmad F. : Application of Crocco-Wang equation to the Blasius problem. Electronic Journal "Technical Acoustics", http://www.ejta.org, 2007, 2.

[4] Brighi B., Fruchard A. and Sari T. : On the Blasius problem. Adv. Differential Equations 13 (2008) 509-600.

[5] Hashim, I. : Comments on A new algorithm for solving classical Blasius equation by L Wang. Appl. Math. Comput. 176 (2006), 700-703.

[6] Liao S. : An optimal homotopy-analysis approach for strongly nonlinear differential equations. Commun. Nonlinear Sci. Numer. Simulat. 15 (2010) 2003-2016.

[7] Wang L. : A new algorithm for solving classical Blasius equation. Appl. Math. Comput. 157 (2004) 1-9.

\begin{tabular}{|c|c|c|c|c|c|}
\hline $\mathrm{n}$ & $\mathrm{a}_{\mathrm{n}}$ & $\overline{\mathrm{a}}_{\mathrm{n}}$ & $\mathrm{n}$ & $\mathrm{a}_{\mathrm{n}}$ & $\overline{\mathrm{a}}_{\mathrm{n}}$ \\
\hline 1 & 0.4082482904 & & 9 & 0.4652579362 & 0.4676507986 \\
\hline 2 & 0.4417428349 & 0.4577552302 & 10 & 0.4657907585 & 0.4678862809 \\
\hline 3 & 0.4525762229 & 0.4622040750 & 11 & 0.4662155662 & 0.4680745445 \\
\hline 4 & 0.4576737760 & 0.4643605941 & 12 & 0.4665613553 & 0.4682281446 \\
\hline 5 & 0.4605662859 & 0.4656031165 & 13 & 0.4668477330 & 0.4683556225 \\
\hline 6 & 0.4624036496 & 0.4663999532 & 14 & 0.4670884028 & 0.4684629350 \\
\hline 7 & 0.4636623198 & 0.4669494712 & 15 & 0.4672932121 & 0.4685544197 \\
\hline 8 & 0.4645724830 & 0.4673488358 & 16 & 0.4674694086 & \\
\hline
\end{tabular}

Table 1. Aitken Acceleration $\left(\bar{a}_{n}=a_{n+1}-\frac{\left(a_{n+1}-a_{n}\right)^{2}}{a_{n+1}-2 a_{n}+a_{n-1}}, n \geq 2\right)$

Received: April 12, 2013 